\title{
ASYMPTOTIC COMPLETENESS FOR RELATIVISTIC KINETIC EQUATIONS WITH SHORT-RANGE INTERACTION FORCES*
}

\author{
SEUNG-YEAL HA ${ }^{\dagger}$, YONG DUCK KIM ${ }^{\dagger}, \mathrm{HO} \mathrm{LEE}^{\dagger}$ AND SE EUN NOH
}

\begin{abstract}
We present an $L^{1}$-asymptotic completeness results for relativistic kinetic equations with short range interaction forces. We show that the uniform phase space-time bound for nonlinear terms to the relativistic nonlinear kinetic equations yields the asymptotic completeness of the relativistic kinetic equations. For this space-time bound, we employ dispersive estimates and explicit construction of a Lyapunov functional.
\end{abstract}

Key words. Asymptotic completeness, relativistic kinetic equation, the Vlasov-Yukawa equation, the Klein-Gordon equation.

AMS subject classifications. Primary 35L45; Secondary 70K20, 70K40

1. Introduction. The purpose of this paper is to address an $L^{1}$-asymptotic completeness for the relativistic kinetic equations (in short RKE) with short range particle-particle or particle-field interactions. Consider an ensemble of classical particles moving with a velocity not negligible with respect to the speed of light. We assume the speed of light and the mass of particle to be unity. In this case, hydrodynamic variables for classical relativistic particles can be calculated by the velocity moments of the one-particle distribution function $F=F(x, \xi, t)$ satisfying an integrodifferential equation:

$$
\begin{aligned}
\partial_{t} F+v(\xi) \cdot \nabla_{x} F & =\mathcal{N}\left(F, \nabla_{\xi} F\right), \quad x, \xi \in \mathbb{R}^{3}, \quad t \in \mathbb{R}_{+}, \\
F(x, \xi, 0) & =F^{i n}(x, \xi),
\end{aligned}
$$

where $v(\xi) \equiv \frac{\xi}{\sqrt{1+|\xi|^{2}}}$ is the relativistic velocity corresponding to momentum $\xi$, and $\mathcal{N}\left(F, \nabla_{\xi} F\right)$ denotes the nonlocal collision operator registering particle-particle interactions, or particle-field interactions. When the nonlinear collision term $\mathcal{N}$ is not present, the resulting equation represents the free streaming of the distribution function in phase space:

$$
\begin{aligned}
\partial_{t} F_{0}+v(\xi) \cdot \nabla_{x} F_{0} & =0, \quad x, \xi \in \mathbb{R}^{3}, \quad t \in \mathbb{R}_{+}, \\
F_{0}(x, \xi, 0) & =\tilde{F}^{i n}(x, \xi),
\end{aligned}
$$

The RKE (1.1) reveals the competitions between "free streaming" and "nonlinearity". Therefore the resulting dynamics of (1.1) is determined by the relative strength of these two competing mechanisms.

Asymptotic completeness for the perturbed system (1.1) refers to the situation that globally perturbed solutions to (1.1) approach to free solutions to (1.2) timeasymptotically. Scattering theory including asymptotic completeness compares the behavior of solutions in the distant future and past of a system evolving in time. In last three decades, potential scattering theory has been extensively studied for classical and quantum $N$-particle systems, PDEs in quantum equations such as Yang-Mill's

\footnotetext{
${ }^{*}$ Received December 1, 2007; accepted for publication April 8, 2008.

$\dagger$ Department of Mathematical Sciences, Seoul National University, Seoul 151-747, Korea (\{syha; dikky; holee; senoh\}@snu.ac.kr). The authors were partially supported by the grants of KOSEF R01-2006-000-10002-0 and BK21-SNU grant.
} 
equation, Schrödinger type equations, the Klein-Gordon equation (see [17, 25, 31] for detailed summary). For the classical and quantum $N$-particle system, the potential scattering is now well established (see [11]) and happens only when the interaction forces are short-ranged. However for particle systems with long-range forces like a Coloumb's force, the standard scattering does not hold. Then the natural question to arise is whether the Vlasov type equations which correspond to the mean-field limit of interacting particle systems show similar scattering behaviors or not. To author's knowledge, this issue has not been much addressed in the previous literatures. We refer to $[21,22,33]$ for the corresponding issue to the linearized Boltzmann equation. Since the full-time rigorous derivation of the Vlasov equation from the interacting particle system is not complete [23], the scattering picture for Vlasov type equations is also not complete.

One of the motivation of this paper is to address the scattering issue from the kinetic level directly without resorting to particle systems. In order to fix the idea, we restrict ourselves to the two relativistic kinetic equations with short range interactions: the relativistic Boltzmann equation and the relativistic Vlasov-Yukawa system.

The rest of this paper is organized as follows. In Section 2, we present an $L^{1}$-asymptotic completeness framework and provide a sufficient condition for $L^{1}$ asymptotic completeness. In Section 3, we study the asymptotic completeness for the relativistic Vlasov-Yukawa system which is a short-range correction of the relativistic Vlasov-Poisson system. Finally Section 4 is devoted to the relativistic Boltzmann equation with constant cross sections. We present a relativistic counterpart for the collision potential measuring all possible future collisions between particles, and as a byproduct of the time-decay estimate of this functional, we obtain the $L^{1}$-asymptotic completeness of the relativistic Boltzmann equation.

Notations. Throughout the paper, $C$ denotes a generic positive constant independent of $t$ and $m$, and we use simplified notations for local and global norms: For any measurable functions $h=h(x, \xi, t)$ and $g=g(x, t)$ defined on $\mathbb{R}_{x}^{3} \times \mathbb{R}_{\xi}^{3} \times \mathbb{R}_{t}$ and $\mathbb{R}_{x}^{3} \times \mathbb{R}_{t}$ respectively, for $1 \leq p \leq \infty$,

$$
\begin{aligned}
& \|h(t)\|_{L_{x, \xi}^{p}}=\|h(\cdot, \cdot, t)\|_{L^{p}\left(\mathbb{R}_{x}^{3} \times \mathbb{R}_{\xi}^{3}\right)}, \quad\|h(x, t)\|\left\|_{L_{\xi}^{p}}=\right\| h(x, \cdot, t) \|_{L^{p}\left(\mathbb{R}_{\xi}^{3}\right)}, \\
& \|h(t)\|_{L_{x}^{\infty}\left(L_{\xi}^{p}\right)}=\sup _{x \in \mathbb{R}^{3}}\|h(x, \cdot, t)\|_{L^{p}\left(\mathbb{R}_{\xi}^{3}\right)}, \quad\|g(t)\|\left\|_{L_{x}^{p}}=\right\| g(\cdot, t)\|\|_{L^{p}\left(\mathbb{R}_{x}^{3}\right)} .
\end{aligned}
$$

2. Asymptotic completeness framework in $L^{1}$. In this section, we present one sufficient condition to the asymptotic completeness for the RKE (1.1).

We now briefly explain several concepts for solutions and basic terminologies in nonlinear scattering theory $[25,34]$. We introduce auxiliary functions denoting quantities evaluated along the free particle trajectory:

$$
\begin{aligned}
& F^{\sharp}(x, \xi, t) \equiv F(x+t v(\xi), \xi, t), \\
& \mathcal{N}\left(F, \nabla_{\xi} F\right)^{\sharp}(x, \xi, t)=\mathcal{N}\left(F, \nabla_{\xi} F\right)(x+t v(\xi), \xi, t) .
\end{aligned}
$$

Then along the particle trajectory, the equation (1.1) can be rewritten as a mild form:

$$
F^{\sharp}(x, \xi, t)=F^{i n}(x, \xi)+\int_{0}^{t} \mathcal{N}\left(F, \nabla_{\xi} F\right)^{\sharp}(x, \xi, s) d s \quad t \geq 0 .
$$

The definition of mild and classical solutions can be stated as follows.

Definition 2.1. (i) Let $T$ be a given positive number. A nonnegative function $F \in C\left([0, T) ; L_{+}^{1}\left(\mathbb{R}^{3} \times \mathbb{R}^{3}\right)\right)$ is a mild solution of $(1.1)$ with a nonnegative initial datum 
$F^{\text {in }}$ if and only if for all $t \in[0, T)$ and a.e $(x, \xi) \in \mathbb{R}^{3} \times \mathbb{R}^{3}, F$ satisfies the integral equation (2.1) pointwise.

(ii) A function $F=F(x, \xi, t) \in C^{1}\left(\mathbb{R}^{3} \times \mathbb{R}^{3} \times[0, T)\right)$ is a classical solution of (1.1) with a nonnegative initial datum $F^{\text {in }}$ if and only if $F$ satisfies the equation (1.1) pointwise.

Below, we restrict ourselves to the situation where the Cauchy problem (1.1) is globally well-posed so that we can address the question of describing and classifying the asymptotic behavior of global-in-time solutions. We introduce solution operators related to the equation (1.1) and (1.2):

$$
\begin{gathered}
U(t), U_{0}(t): \text { solution operators for the nonlinear flow (1.1) } \\
\text { and free flow (1.2) respectively. }
\end{gathered}
$$

Then $\{U(t)\}$ satisfies a one-parameter semi-group property of operator:

$$
U(t) U(s)=U(t+s), \quad U(0)=I, \quad t, s \in[0, \infty),
$$

and solutions to (1.1) and (1.2) can be formally represented as follows:

$$
F(t)=U(t) F^{i n}, \quad F_{0}(t)=U_{0}(t) \tilde{F}^{i n}, \quad t \geq 0 .
$$

We define the "asymptotic completeness" to (1.1) as follows.

Definition 2.2. The nonlinear equation (1.1) satisfies "asymptotic completeness" in $L^{1}$ if and only if for every global solution $F$ to (1.1), there exists asymptotic state $F_{ \pm}$satisfying

$$
\lim _{t \rightarrow \pm \infty}\left\|U(t) F^{i n}-U_{0}(t) F_{ \pm}\right\|_{L_{x, \xi}^{1}}=0 .
$$

REMARK 2.3. The scattering operator $\mathcal{S}$ is defined as the operator $F_{-} \rightarrow F_{+}$ acting between pairs of free states, and the wave operators $W_{+}$and $W_{-}$are the operators $F_{ \pm} \rightarrow F$, acting from free states to interacting states.

We next present a sufficient condition for the time-forward asymptotic completeness condition:

$$
\begin{aligned}
& \int_{0}^{\infty} \mathcal{N}\left(F, \nabla_{\xi} F\right)^{\sharp}(x, \xi, t) d t<\infty, \quad \text { a. e. }(x, \xi) \in \mathbb{R}^{6}, \\
& \int_{0}^{\infty} \int_{\mathbb{R}^{6}}\left|\mathcal{N}\left(F, \nabla_{\xi} F\right)^{\sharp}(x, \xi, t)\right| d \xi d x d t<\infty .
\end{aligned}
$$

Proposition 2.1. Let $F$ be a global mild solution in $C\left(\mathbb{R}^{+} ; L^{1}\left(\mathbb{R}^{3} \times \mathbb{R}^{3}\right)\right)$ to (1.1) with initial datum $F^{\text {in }}$. Suppose $F$ satisfies the scattering condition (2.2). Then there exists the unique $L^{1}$-scattering state $F_{+} \in L_{+}^{1}\left(\mathbb{R}^{6}\right)$ such that

$$
\lim _{t \rightarrow \infty}\left\|U(t) F^{i n}-U_{0}(t) F_{+}\right\|_{L_{x, \xi}^{1}}=0 .
$$

Proof. Let $F$ be a global mild solution in $C\left(\mathbb{R}^{+} ; L^{1}\left(\mathbb{R}^{3} \times \mathbb{R}^{3}\right)\right)$ to (1.1) with initial datum $F^{\text {in }}$. Then we have

$$
F^{\sharp}(x, \xi, t)=F^{i n}(x, \xi)+\int_{0}^{t} \mathcal{N}\left(F, \nabla_{\xi} F\right)^{\sharp}(x, \xi, s) d s \quad t \geq 0 .
$$


We set the asymptotic scattering state $F_{+}$by

$$
F_{+}(x, \xi) \equiv F^{i n}(x, \xi)+\int_{0}^{\infty} \mathcal{N}\left(F, \nabla_{\xi} F\right)^{\sharp}(x, \xi, s) d s .
$$

Then it follows from (2.2) that the asymptotic state $F_{+}$is well-defined a.e. We now subtract (2.4) from (2.3), and take an $L^{1}$-norm to find

$$
\left\|F^{\sharp}(t)-F_{+}\right\|_{L_{x, \xi}^{1}} \leq \int_{t}^{\infty} \int_{\mathbb{R}^{6}}\left|\mathcal{N}\left(F, \nabla_{\xi} F\right)^{\sharp}(x, \xi, s)\right| d \xi d x d s .
$$

We let $t \rightarrow+\infty$ and use the scattering condition (2.2) to conclude

$\lim _{t \rightarrow+\infty}\left\|F^{\sharp}(t)-F_{+}\right\|_{L_{x, \xi}^{1}}=0, \quad$ or equivalently $\quad \lim _{t \rightarrow+\infty}\left\|U(t) F^{i n}-U_{0}(t) F_{+}\right\|_{L_{x, \xi}^{1}}=0$.

REMARK 2.4. 1. For the non-relativistic Boltzmann equation, asymptotic completeness in $L^{\infty}$ and $L^{1}$-norms are well studied in previous literatures [18, 22, 26, 32, $35]$.

2. For the relativistic Vlasov-Poisson system, the $L_{x, \xi}^{1}$-norm of the nonlinear term $\mathcal{N}\left(F, \nabla_{\xi} F\right)=\nabla_{x} \varphi \cdot \nabla_{\xi} F$ is not integrable in $t$. More precisely, for small and decaying classical solutions in $[3,4,8]$, we have

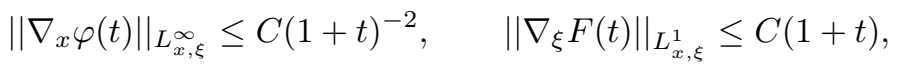

These estimates yield

$$
\left|\int_{\mathbb{R}^{6}} \nabla_{x} \varphi \cdot \nabla_{\xi} F(x, \xi, t) d \xi d x\right| \leq\left. C|| \nabla_{x} \varphi(t)\right|_{L_{x, \xi}^{\infty}}|| \nabla_{\xi} F(t) \|_{L_{x, \xi}^{1}} \leq C(1+t)^{-1} .
$$

Hence Proposition 2.1 is not applicable for the Vlasov-Poisson system in three dimensions.

In the following two sections, as an application of Proposition 2.1, we consider two relativistic kinetic equations with short-range interactions: the Vlasov-Yukawa system and the Boltzmann equation. For the Vlasov-Yukawa system, we use direct dispersion estimates to prove that small classical solutions satisfy the asymptotic completeness condition (2.2). In contrast, for the relativistic Boltzmann equation, since the pointwise estimates for solutions are not available yet, we instead employ a detour route to get (2.2) via a Lyapunov functional in a priori setting.

3. The relativistic Vlasov-Yukawa equation. In this section, we consider the ensemble of classical particles moving with a velocity not negligible with respect to the speed of light and interacting with a scalar Yukawa field [36]. In this case the dynamics of the distribution function $F$ is well described by relativistic Vlasov equation coupled with the scalar Yukawa equation [7, 20]:

$$
\begin{aligned}
& \partial_{t} F+v(\xi) \cdot \nabla_{x} F-\nabla_{x} \varphi_{Y} \cdot \nabla_{\xi} F=0, \quad x, \xi \in \mathbb{R}^{3}, \quad t>0, \\
& \varphi_{Y}(x, t)=\frac{\nu}{4 \pi} \int_{\mathbb{R}^{3}} \frac{e^{-m|x-y|}}{|x-y|} \rho(y, t) d y, \quad \rho \equiv \int_{\mathbb{R}^{3}} f d \xi,
\end{aligned}
$$

with initial datum

$$
F(x, \xi, 0)=F^{i n}(x, \xi), \quad x, \xi \in \mathbb{R}^{3},
$$


where $\nu$ is a constant representing the nature of interaction force, say $\nu=1$ for repulsive force, $\nu=-1$ for attractive force, and $m$ denotes the mass of a force carrier particle (e.g. pions in proton-neutron interactions).

The global existence of classical solutions to the non-relativistic system (3.1) with unbounded mass was first considered by Caprino, Marchioro and Pulvirenti [7] in two dimensions, whereas the small global classical solutions with finite mass in three dimensions was recently studied by Ha and Lee [20] employing the standard arguments in Vlasov theory [30]. The system (3.1) can be viewed as a short-range correction of the relativistic Vlasov-Poisson system:

$$
\begin{aligned}
& \partial_{t} F+v(\xi) \cdot \nabla_{x} F-\nabla_{x} \varphi_{C} \cdot \nabla_{\xi} F=0, \quad x, \xi \in \mathbb{R}^{3}, \quad t>0, \\
& \varphi_{C}(x, t)=\frac{\nu}{4 \pi} \int_{\mathbb{R}^{3}} \frac{\rho(y, t)}{|x-y|} d y, \quad \rho \equiv \int_{\mathbb{R}^{3}} f d \xi
\end{aligned}
$$

As $m \rightarrow 0$, classical solutions of the Vlasov-Yukawa system converge to the corresponding solutions of the Vlasov-Poisson system a.e. (see [20] for detailed proof). Below, we cite the global existence theorem in [20] for classical solutions with small data without a proof.

LEMma 3.1. [20] Suppose the nonnegative initial datum $F^{i n} \in C^{1}\left(\mathbb{R}^{6}\right)$ has a compact support in $x$ and $\xi$, and is small in $W^{1, \infty}$-norm, more precisely,

$$
\sum_{0 \leq|\alpha|+|\beta| \leq 1}\left\|\nabla_{x}^{\alpha} \nabla_{\xi}^{\beta} f^{i n}\right\|_{L_{x, \xi}^{\infty}}<\delta \ll 1,
$$

Then the Vlasov-Yukawa system (3.1) has a global-in-time classical solution, and the solution and force $F_{Y}=-\nabla_{x} \varphi_{Y}$ satisfy the following uniform estimates.

$$
\begin{aligned}
& \|F(t)\|_{L_{x, \xi}^{\infty}} \leq \delta, \quad\|F(t)\|_{L_{x, \xi}^{1}} \leq C \delta, \quad\|F(t)\|_{L_{x}^{\infty}\left(L_{\xi}^{1}\right)} \leq \frac{C \delta}{(1+t)^{3}}, \\
& \left\|\nabla_{\xi} F(t)\right\|_{L_{x, \xi}^{1}} \leq C \delta(1+t), \quad\left\|\nabla_{\xi} F(t)\right\|_{L_{x}^{\infty}\left(L_{\xi}^{1}\right)} \leq \frac{C \delta}{(1+t)^{2}}, \\
& \left\|\nabla_{x}^{k+1} \varphi_{Y}(t)\right\|_{L_{x}^{\infty}}+\left\|\partial_{t} \nabla_{x} \varphi_{Y}(t)\right\|_{L_{x}^{\infty}} \leq \frac{C \delta}{m(1+t)^{3}} . \quad k=0,1,2 .
\end{aligned}
$$

REMARK 3.2. For the dispersion type estimates for the Vlasov-Poisson system, we refer to $[9,10,29]$.

The asymptotic completeness of the Vlasov-Yukawa system follows from Lemma 3.1 and Proposition 2.1.

THEOREM 3.3. Let $F$ be a classical solution to (3.1) with initial datum $F^{\text {in }}$. Then there exists the unique scattering state $F_{+}$such that

$$
\lim _{t \rightarrow \infty}\left\|U(t) F^{i n}-U_{0}(t) F_{+}\right\|_{L_{x, \xi}^{1}}=0
$$

Proof. It suffices to show that $F$ satisfies the asymptotic completeness condition $(2.2)$.

(i) Since $\mathcal{N}\left(F, \nabla_{\xi} F\right)=-\nabla_{x} \varphi_{Y} \cdot \nabla_{\xi} F$,

$$
\left|\mathcal{N}\left(F, \nabla_{\xi} F\right)\right|(x, \xi, t) \leq\left\|\nabla_{x} \varphi_{Y}\right\|_{L_{x, \xi}^{\infty}}\left\|\nabla_{\xi} F\right\|_{L_{x, \xi}^{\infty}} \leq \frac{C \delta^{2}}{m(1+t)^{2}} .
$$


This verifies the first condition in (2.2).

(ii) Note that

$$
\int_{\mathbb{R}^{6}}\left|\nabla_{x} \varphi_{Y}(x, t) \cdot \nabla_{\xi} F(x, \xi, t)\right| d \xi d x \leq\left\|\nabla_{x} \varphi_{Y}(t)\right\|_{L_{x}^{\infty}}\left\|\nabla_{\xi} F(t)\right\|_{L_{x, \xi}^{1}} \leq \frac{C \delta^{2}}{m(1+t)^{2}} .
$$

We now integrate the above inequality to get

$$
\int_{0}^{\infty} \int_{\mathbb{R}^{6}}\left|\nabla_{x} \varphi_{Y}(x, t) \cdot \nabla_{\xi} F(x, \xi, t)\right| d \xi d x d t \leq \frac{C \delta^{2}}{m}<\infty .
$$

Hence Proposition 2.1 yields the desired result.

4. The relativistic Boltzmann equation. In this section, we consider the relativistic Boltzmann equation dealing with the spatial-temporal evolution of the distribution function $F$ of identical dilute relativistic classical particles undergoing binary collisions. In the absence of external forces, $F$ satisfies

$$
\begin{aligned}
\partial_{t} F+v(\xi) \cdot \nabla_{x} F & =Q(F, F), \quad x, \xi \in \mathbb{R}^{3}, \quad t>0, \\
F(x, \xi, 0) & =F^{i n}(x, \xi),
\end{aligned}
$$

where $Q(F, F)$ denotes a collision operator which takes binary collisions between particles into account.

Let $\left(\xi, \xi_{*}\right)$ and $\left(\xi^{\prime}, \xi_{*}^{\prime}\right)$ be pairs of pre-collisional and post-collisional momentum respectively, and they satisfy conservation of momentum and energy

$$
\xi+\xi_{*}=\xi^{\prime}+\xi_{*}^{\prime}, \quad \xi_{0}+\xi_{* 0}=\xi_{0}^{\prime}+\xi_{* 0}^{\prime}, \quad \xi_{0}=\sqrt{1+|\xi|^{2}} .
$$

The above relations yield the following collision transformation:

$$
\xi^{\prime}=\xi-a\left(\xi, \xi_{*}, \omega\right) \omega, \quad \xi_{*}^{\prime}=\xi_{*}+a\left(\xi, \xi_{*}, \omega\right) \omega, \quad \omega \in \mathbf{S}^{2},
$$

where

$$
a\left(\xi, \xi_{*}, \omega\right)=\frac{2\left(\xi_{0}+\xi_{* 0}\right)\left[\left(v(\xi)-v\left(\xi_{*}\right)\right) \cdot \omega\right] \xi_{0} \xi_{* 0}}{\left(\xi_{0}+\xi_{* 0}\right)^{2}-\left[\left(\xi+\xi_{*}\right) \cdot \omega\right]^{2}} .
$$

The relativistic collision operator $Q(f, f)$ takes the form of

$$
Q(F, F)(x, \xi, t) \equiv \int_{\mathbb{R}^{3} \times \mathbf{S}_{+}^{2}} \xi_{M} \sigma(g, \theta)\left(F^{\prime} F_{*}^{\prime}-F F_{*}\right) d \omega d \xi_{*},
$$

with the standard handy notations:

$$
F_{*}=F\left(x, \xi_{*}, t\right), \quad F^{\prime}=F\left(x, \xi^{\prime}, t\right) \quad \text { and } \quad F_{*}^{\prime}=F\left(x, \xi_{*}^{\prime}, t\right) .
$$

Here $\sigma=\sigma(g, \theta)$ and $\xi_{M}$ denote the scattering cross section and the Møller velocity respectively. We now introduce some quantities employed in the collision integral. We set

$$
\begin{aligned}
s & \equiv 2\left(\sqrt{1+\left|\xi_{*}\right|^{2}} \sqrt{1+|\xi|^{2}}-\xi_{*} \cdot \xi+1\right), \\
4 g^{2} & \equiv 2\left(\sqrt{1+\left|\xi_{*}\right|^{2}} \sqrt{1+|\xi|^{2}}-\xi_{*} \cdot \xi-1\right) \\
& =s-4
\end{aligned}
$$


The Møller velocity $\xi_{M}$ is defined as

$$
\xi_{M}^{2} \equiv\left|v(\xi)-v\left(\xi_{*}\right)\right|^{2}-\left|v(\xi) \times v\left(\xi_{*}\right)\right|^{2} .
$$

This implies

$$
\xi_{M}^{2}=\frac{s(s-4)}{4 \xi_{0}^{2} \xi_{* 0}^{2}} \quad \text { and } \quad \xi_{M} \leq\left|v(\xi)-v\left(\xi_{*}\right)\right| .
$$

The local existence of mild solutions to (4.1) was studied in $[1,2,5,6]$, and the global existence for space homogeneous problem was treated in [28]. In a closeto-a global Maxwellian regime, the existence theory for the space inhomogeneous relativistic Boltzmann equation is now well established in $[15,16]$, whereas in a closeto-vacuum regime, we do not have satisfactory results yet for the existence of global mild solutions, even if we have some partial results [5, 12, 13, 27]. Recently, Glassey [14] obtained global mild solutions to the relativistic Boltzmann equation near vacuum data employing an interesting function space and the standard Kaniel-Shinbrot argument $[22,24]$ under the rather strong assumption on the collisional cross section $\sigma(g, \theta)$.

Below we define a set $\mathcal{S}_{p q}(\varepsilon, T)$, where we look for a priori asymptotic completeness estimates, and list main framework and assumptions $\mathcal{A}$. For positive constants $p, q, \varepsilon$,

$$
\mathcal{S}_{p q}(\varepsilon, T) \equiv\left\{g \in C\left(\mathbb{R}^{3} \times \mathbb{R}^{3} \times[0, T)\right)\left|\|g\| \equiv \sup _{x, \xi, t}\right| g^{\sharp}(x, \xi, t) \mid e^{p|x|^{2}} e^{q \sqrt{1+|\xi|^{2}}}<\varepsilon\right\},
$$

and main assumptions are as follows.

- $\mathcal{A} 1$ : The differential cross section $\sigma(g, \theta)$ in (4.2) is bounded, i.e.,

$$
\sigma(g, \theta) \leq \sigma^{*}<\infty .
$$

- $\mathcal{A} 2$ : The size $\varepsilon$ of classical solutions satisfies

$$
0<\varepsilon<\frac{q^{3}}{32 \pi^{2} \sigma^{*}} \sqrt{\frac{p}{\pi}}
$$

REMARK 4.1. (1) For the non-relativistic Boltzmann equation with the velocitymomentum relation $v(\xi)=\xi$, the existence of global mild solutions to the nonrelativistic Boltzmann equation:

$$
\partial_{t} F+\xi \cdot \nabla_{x} F=Q(F, F),
$$

is well established in the set $\mathcal{S}_{p q}(\varepsilon, \infty)$ with $\varepsilon \ll 1$ (see Glassey's book [14]). In fact, the non-relativistic Boltzmann equation is globally well-posed in the class of algebraically decaying solutions (see $[18,22,32]$ ). In contrast for the relativistic Boltzmann equation, it is not known whether we can find the global mild solutions in the set $\mathcal{S}_{p q}(\varepsilon, \infty)$.

(2) The bounded differential cross section in $\mathcal{A} 1$ has been employed in previous literatures $[5,6]$.

We next explicitly construct a Lyapunov functional which yields the scattering condition (2.2). Define the relativistic collision potential $\mathcal{D}$ and the collision production rate $\Lambda$ along the classical solution $F$ :

$$
\mathcal{D}(F(t)) \equiv \int_{\mathbb{R}^{6}} F^{\sharp}(x, \xi, t)
$$




$$
\begin{aligned}
& \times\left[\int_{\mathbb{R}^{3}} \int_{0}^{\infty}\left|v(\xi)-v\left(\xi_{*}\right)\right| F^{\sharp}\left(x+(t+\tau)\left(v(\xi)-v\left(\xi_{*}\right)\right), \xi_{*}, t\right) d \tau d \xi_{*}\right] d \xi d x, \\
\Lambda(F(t)) & \equiv \int_{\mathbb{R}^{9}}\left|v(\xi)-v\left(\xi_{*}\right)\right| F^{\sharp}(x, \xi, t) F^{\sharp}\left(x+t\left(v(\xi)-v\left(\xi_{*}\right)\right), \xi_{*}, t\right) d \xi_{*} d \xi d x .
\end{aligned}
$$

The positive quantity inside the bracket in $\mathcal{D}(F(t))$ denotes the total number of particles to collide with test particles $F^{\sharp}(x, \xi, t)$ in future. Note that

$$
\begin{aligned}
\int_{\mathbb{R}^{6}} Q_{-}^{\sharp} & (F, F)(x, \xi, t) d \xi d x \\
& =\int_{\mathbb{R}^{9} \times \mathbf{S}_{+}^{2}} \xi_{M} \sigma F(x+t v(\xi), \xi, t) F\left(x+t v(\xi), \xi_{*}, t\right) d \omega d \xi_{*} d \xi d x \\
& \leq 2 \pi \sigma^{*} \int_{\mathbb{R}^{9}}\left|v(\xi)-v\left(\xi_{*}\right)\right| F^{\sharp}(x, \xi, t) F^{\sharp}\left(x+t\left(v(\xi)-v\left(\xi_{*}\right)\right), \xi_{*}, t\right) d \xi_{*} d \xi d x \\
& =2 \pi \sigma^{*} \Lambda(F(t)) .
\end{aligned}
$$

Since $\int Q^{\sharp}(F, F) d \xi d x=0$, we have

$$
\int_{\mathbb{R}^{6}} Q_{+}^{\sharp}(F, F)(x, \xi, t) d \xi d x=\int_{\mathbb{R}^{6}} Q_{-}^{\sharp}(F, F)(x, \xi, t) d \xi d x \leq 2 \pi \sigma^{*} \Lambda(F(t)) .
$$

Next lemma establishes that the collision potential in $\mathcal{S}_{p q}(\varepsilon, \infty)$ is well-defined.

Lemma 4.2. Suppose the main assumptions $\mathcal{A} 1-\mathcal{A} 2$ hold, and let $F$ be a classical solution satisfying $\|F\| \leq \varepsilon$. Then we have

$$
\int_{\mathbb{R}^{3}} \int_{0}^{\infty}\left|v(\xi)-v\left(\xi_{*}\right)\right| F^{\sharp}\left(x+(t+\tau)\left(v(\xi)-v\left(\xi_{*}\right)\right), \xi_{*}, t\right) d \tau d \xi_{*} \leq \frac{8 \pi}{q^{3}} \sqrt{\frac{\pi}{p}} \varepsilon .
$$

Proof. Since $\|F\| \leq \varepsilon$, we have

$$
F^{\sharp}\left(x+(t+\tau)\left(v(\xi)-v\left(\xi_{*}\right)\right), \xi_{*}, t\right) \leq \varepsilon e^{-p\left|x+(t+\tau)\left(v(\xi)-v\left(\xi_{*}\right)\right)\right|^{2}} e^{-q \sqrt{1+\left|\xi_{*}\right|^{2}}} .
$$

Hence we use the above inequality to see

$$
\begin{aligned}
\int_{\mathbb{R}^{3}} & \int_{0}^{\infty}\left|v(\xi)-v\left(\xi_{*}\right)\right| F^{\sharp}\left(x+(t+\tau)\left(v(\xi)-v\left(\xi_{*}\right)\right), \xi_{*}\right) d \tau d \xi_{*} \\
& \leq \varepsilon \int_{\mathbb{R}^{3}}\left|v(\xi)-v\left(\xi_{*}\right)\right| e^{-q \sqrt{1+\left|\xi_{*}\right|^{2}}}\left(\int_{0}^{\infty} e^{-p\left|x+(t+\tau)\left(v(\xi)-v\left(\xi_{*}\right)\right)\right|^{2}} d \tau\right) d \xi_{*} \\
& \leq \varepsilon \sqrt{\frac{\pi}{p}} \int_{\mathbb{R}^{3}} e^{-q \sqrt{1+\left|\xi_{*}\right|^{2}}} d \xi_{*} \\
& \leq \varepsilon \sqrt{\frac{\pi}{p}} \int_{\mathbb{R}^{3}} e^{-q\left|\xi_{*}\right|} d \xi_{*} \\
& \leq \frac{8 \pi \varepsilon}{q^{3}} \sqrt{\frac{\pi}{p}}
\end{aligned}
$$

where we used

$$
\int_{\mathbb{R}_{+}} e^{-p\left|x+(t+\tau)\left(v(\xi)-v\left(\xi_{*}\right)\right)\right|^{2}} d \tau \leq \sqrt{\frac{\pi}{p}} \frac{1}{\left|v(\xi)-v\left(\xi_{*}\right)\right|} .
$$


REMARK 4.3. The collision potential $\mathcal{D}(F(t))$ is a priori bounded by the total mass.

$$
|\mathcal{D}(F(t))| \leq \frac{8 \pi \varepsilon}{q^{3}} \sqrt{\frac{\pi}{p}}\left\|F^{i n}\right\|_{L_{x, \xi}^{1}} .
$$

We next show the collision potential is a Lyapunov functional.

Lemma 4.4. Suppose the main assumptions $\mathcal{A} 1-\mathcal{A} 2$ hold, and let $F$ be a classical solution in $\mathcal{S}_{p q}(\varepsilon, \infty)$ corresponding initial datum $F^{i n}$. Then the collision potential $\mathcal{D}(F)$ satisfies

$$
\mathcal{D}(F(t))+C_{0} \int_{0}^{t} \Lambda(F(s)) d s \leq \mathcal{D}\left(F^{i n}\right), \quad t \geq 0
$$

where $C_{0}$ is a positive constant independent of time $t$.

Proof. It follows from (4.1) that

$$
\begin{aligned}
& \partial_{t} F^{\sharp}(x, \xi, t)=Q^{\sharp}(F, F)(x, \xi, t), \\
& \partial_{t}\left[F^{\sharp}\left(x+(t+\tau)\left(v(\xi)-v\left(\xi_{*}\right)\right), \xi_{*}, t\right)\right] \\
& =\partial_{t}\left[F\left(x+t v(\xi)+\tau\left(v(\xi)-v\left(\xi_{*}\right)\right), \xi_{*}, t\right)\right] \\
& =\left(\partial_{t} F+v(\xi) \cdot \nabla_{x} F\right)\left(x+t v(\xi)+\tau\left(v(\xi)-v\left(\xi_{*}\right)\right), \xi_{*}, t\right) \\
& =\left(v(\xi)-v\left(\xi_{*}\right)\right) \cdot \nabla_{x} F^{\sharp}\left(x+(t+\tau)\left(v(\xi)-v\left(\xi_{*}\right)\right), \xi_{*}, t\right) \\
& +Q^{\sharp}(F, F)\left(x+(t+\tau)\left(v(\xi)-v\left(\xi_{*}\right)\right), \xi_{*}, t\right) \text {. }
\end{aligned}
$$

The first term in the right hand side of (4.4) can be rewritten as

$$
\begin{gathered}
\left(v(\xi)-v\left(\xi_{*}\right)\right) \cdot \nabla_{x} F^{\sharp}\left(x+(t+\tau)\left(v(\xi)-v\left(\xi_{*}\right)\right), \xi_{*}, t\right) \\
=\partial_{\tau}\left[F^{\sharp}\left(x+(t+\tau)\left(v(\xi)-v\left(\xi_{*}\right)\right), \xi_{*}, t\right)\right] .
\end{gathered}
$$

We use the above relation to see

$$
\begin{aligned}
\partial_{t} & \left(\left|v(\xi)-v\left(\xi_{*}\right)\right| F^{\sharp}(x, \xi, t) F^{\sharp}\left(x+(t+\tau)\left(v(\xi)-v\left(\xi_{*}\right)\right), \xi_{*}, t\right)\right) \\
& =\partial_{\tau}\left(\left|v(\xi)-v\left(\xi_{*}\right)\right| F^{\sharp}(x, \xi, t) F^{\sharp}\left(x+(t+\tau)\left(v(\xi)-v\left(\xi_{*}\right)\right), \xi_{*}, t\right)\right) \\
& +\left|v(\xi)-v\left(\xi_{*}\right)\right| Q^{\sharp}(F, F)(x, \xi, t) F^{\sharp}\left(x+(t+\tau)\left(v(\xi)-v\left(\xi_{*}\right)\right), \xi_{*}, t\right) \\
& +\left|v(\xi)-v\left(\xi_{*}\right)\right| F^{\sharp}(x, \xi, t) Q^{\sharp}(F, F)\left(x+(t+\tau)\left(v(\xi)-v\left(\xi_{*}\right)\right), \xi_{*}, t\right) .
\end{aligned}
$$

Since the integrand and its $\partial_{t}$ derivative inside $\mathcal{D}(F(t))$ are continuous, and $\int \partial_{t}(\cdots)$ exists, we can interchange time-derivative and integral, when we perform timederivative of $\mathcal{D}(F(t))$. We integrate (4.5) over $\mathbb{R}^{3} \times \mathbb{R}^{3} \times \mathbb{R}^{3} \times \mathbb{R}_{+}$with respect 
to $\left(x, \xi, \xi_{*}, \tau\right)$ to get

$$
\begin{aligned}
\frac{d \mathcal{D}(F(t))}{d t} & =-\int_{\mathbb{R}^{9}}\left|v(\xi)-v\left(\xi_{*}\right)\right| F^{\sharp}(x, \xi, t) F^{\sharp}\left(x+t\left(v(\xi)-v\left(\xi_{*}\right)\right), \xi_{*}, t\right) d \xi_{*} d \xi d x \\
& +\int_{\mathbb{R}^{9} \times \mathbb{R}_{+}}\left|v(\xi)-v\left(\xi_{*}\right)\right| Q^{\sharp}(F, F)(x, \xi, t) \\
& \times F^{\sharp}\left(x+(t+\tau)\left(v(\xi)-v\left(\xi_{*}\right)\right), \xi_{*}, t\right) d \tau d \xi_{*} d \xi d x \\
& +\int_{\mathbb{R}^{9} \times \mathbb{R}_{+}}\left|v(\xi)-v\left(\xi_{*}\right)\right| Q^{\sharp}(F, F)\left(x+(t+\tau)\left(v(\xi)-v\left(\xi_{*}\right)\right), \xi_{*}, t\right) \\
& \times F^{\sharp}(x, \xi, t) d \tau d \xi_{*} d \xi d x \\
& \equiv-\Lambda(F(t))+\mathcal{I}_{1}(t)+\mathcal{I}_{2}(t) .
\end{aligned}
$$

By the change of variable $\left(x+(t+\tau)\left(v(\xi)-v\left(\xi_{*}\right)\right) \rightarrow \bar{x}\right)$, we have $\mathcal{I}_{1}=\mathcal{I}_{2}$. Now we use Lemma 2 to estimate $\mathcal{I}_{1}$

$$
\begin{aligned}
\mathcal{I}_{1}(t) & \leq \int_{\mathbb{R}^{6}} Q_{+}^{\sharp}(F, F)(x, \xi) \\
& \times\left[\int_{\mathbb{R}^{3} \times \mathbb{R}_{+}}\left|v(\xi)-v\left(\xi_{*}\right)\right| F^{\sharp}\left(x+(t+\tau)\left(v(\xi)-v\left(\xi_{*}\right)\right), \xi_{*}, t\right) d \tau d \xi_{*}\right] d \xi d x \\
& \leq \frac{16 \pi^{2} \sigma^{*}}{q^{3}} \sqrt{\frac{\pi}{p}} \varepsilon \Lambda(F(t)) .
\end{aligned}
$$

In (4.6), we use (4.7) and $\mathcal{I}_{1}=\mathcal{I}_{2}$ to see

$$
\begin{aligned}
\frac{d \mathcal{D}(F(t))}{d t} & \leq\left(-1+\frac{32 \pi^{2} \sigma^{*}}{q^{3}} \sqrt{\frac{\pi}{p}} \varepsilon\right) \Lambda(F(t)) \\
& \leq-C_{0} \Lambda(F(t)),
\end{aligned}
$$

where $C_{0}$ is a positive constant independent of time $t$. Finally we integrate (4.8) with respect to $t$ to get the desired result.

Proposition 4.1. Let $F$ be a classical solution in $\mathcal{S}_{p q}(\varepsilon, \infty)$ with initial datum $F^{\text {in }}$, moreover assume that $F$ satisfies

$$
\int_{0}^{\infty} Q_{+}^{\sharp}(F, F)(x, \xi, t) d t<\infty, \quad(x, \xi) \in \mathbb{R}^{6} .
$$

Then there exists the unique scattering state $F_{+}$such that

$$
\lim _{t \rightarrow \infty}\left\|U(t) F^{i n}-U_{0}(t) F_{+}\right\|_{L_{x, \xi}^{1}}=0 .
$$

Proof. Let $F$ be a classical solution in $\mathcal{S}_{p q}(\varepsilon, \infty)$ with initial datum $F^{\text {in }}$ satisfying

$$
\int_{0}^{\infty} Q_{+}^{\sharp}(F, F)(x, \xi, t) d t<\infty, \quad(x, \xi) \in \mathbb{R}^{6} .
$$

We now need to check scattering condition (2.2) to prove the main result.

(i) We follows the procedure of the pointwise estimate of $Q_{-}^{\sharp}(F, F)$ given in $[18,19]$ to get

$$
\left|Q_{-}^{\sharp}(F, F)\right|(x, \xi, t) \leq \frac{C\left(\varepsilon, \sigma^{*}, q\right) e^{-p|x|^{2}} e^{-q \sqrt{1+|\xi|^{2}}}}{(1+t)^{3}} .
$$


This yields

$$
\int_{0}^{\infty} Q_{-}^{\sharp}(F, F)(x, \xi, t) d t<\infty .
$$

On the other hand, the pointwise estimate for $Q_{+}^{\sharp}(F, F)$ is not available yet. This is one of the main reason that we do not have a global existence in $\mathcal{S}_{p q}(\varepsilon, \infty)$. We now combine the assumption (4.9) and (4.10) to check the first condition in (2.2).

(ii) We now check the second condition in asymptotic completeness criterion (2.2). The second condition follows from the Lemma 4.4:

$$
\int_{0}^{\infty} \int_{\mathbb{R}^{6}}\left|Q^{\sharp}(F, F)(x, \xi, t)\right| d x d \xi d t \leq 4 \pi \sigma^{*} \int_{0}^{\infty} \Lambda(F(t)) d t \leq \frac{4 \pi \sigma^{*}}{C_{0}} \mathcal{D}\left(F^{i n}\right)<\infty .
$$

Then Proposition 2.1 implies the desired result. $\square$

5. Conclusion. In this paper, we have presented a sufficient condition for the $L^{1}$-asymptotic completeness to the relativistic kinetic equations with short-range interactions, and as a direct application of this condition, we showed that the relativistic Vlasov-Yukawa system and the relativistic Boltzmann equation with a bounded differential cross section satisfy asymptotic-completeness in suitable solution spaces. To the authors' knowledge, the $L^{1}$-asymptotic completeness problem for Vlasov equations with long-range interaction forces have not been not addressed in previous literatures, and we think that this problem is an interesting open problem in kinetic scattering theory.

\section{REFERENCES}

[1] Bancel, D., Problème de Cauchy pour l'équation de Boltzmann en relativité générale, Ann. Inst. Henri Poincareé, XVIII 3 (1973), pp. 263-284.

[2] Bancel, D., Choquet-Bruhat, Y., Uniqueness and local stability for the Einstein-MaxwellBoltzmann system, Comm. Math. Phys., 33 (1973), pp. 83-96.

[3] Bardos, C., Ngonn, H. T., Degond, P., Existence globale des solutions des équations de Vlasov-Poisson relativistes en dimension 3, C.R. Acad. Sci. Paris., 301 (1985), pp. 265268.

[4] Bardos, C., Degond, P., Global existence for the Vlasov-Poisson equation in three space variables with small initial data, Ann. Inst. Henri Poincare, Analyse non lineaire, 2 (1985), pp. 101-118.

[5] Bichteler, K., On the Cauchy problem for relativistic Boltzmann equation, Comm. Math. Phys., 4 (1967), pp. 352-364.

[6] Calogero, S., The Newtonian limit of the relativistic Botlzmann equation, J. Math. Phys., 45 (2004), pp. 4042-4052.

[7] Caprino, S., Marchioro, C., Pulvirenti, M., On the two-dimensional Vlasov-Helmholtz equation with infinite mass, CPDE, 27 (2002), pp. 791-808.

[8] Chae, M., Ha, S.-Y., New Lyapunov functionals of the Vlasov-Poisson system, SIAM J. Math. Anal., 37 (2006), pp. 1709-1731.

[9] Dolbeault, J., Fernandez, J., Sanchez, O., Stability for the gravitational Vlasov-Poisson system in dimension two, Comm. Partial Differential Equations, 31 (2006), pp. 1425-1449.

[10] Dolbeault, J., Sanchez, O., Soler, J., Asymptotic behaviour for the Vlasov-Poisson system in the stellar-dynamics case, Arch. Ration. Mech. Anal., 171 (2004), pp. 301-327.

[11] Dereziński, J., Gerard, C., Scattering theory of classical and quantum N-particle systems, Texts and Monographs in Physics, Springer-Verlag, Berlin, 1997.

[12] Dudyński, M., Ekiel Jezewska, M., On the linearized Relativistic Boltzmann equation, Comm. Math. Phys., 115 (1988), pp. 607-629.

[13] Dudyński, M., Ekiel Jezewska, M., Global existence proof for relativistic Boltzmann equation, J. Stat. Phys., 66 (1992), pp. 991-1001. 
[14] Glassey, R. T., Global solutions to the Cauchy problem for the relativistic Boltzmann equation with near-vacuum data, Comm. Math. Phys., 264 (2006), pp. 705-724.

[15] Glassey, R. T., Strauss, W., Asymptotic stability of the relativistic Maxwellian, Publ. R.I.M.S. Kyoto Univ., 29 (1993), pp. 301-347.

[16] Glassey, R. T., Strauss, W., Asymptotic stability of the relativistic Maxwellian via fourteen moments, Trans. Th. Stat. Phys., 24 (1995), pp. 657-678.

[17] Glassey, R. T., Strauss, W., The scattering of certain Yang-Mills fields, Comm. Math. Phys., 89 (1983), pp. $465-482$.

[18] HA, S.-Y., Nonlinear functionals of the Boltzmann equation and uniform stability estimates, J. Differential Equations, 215 (2005), pp. 178-205.

[19] HA, S.-Y., $L^{1}$ stability of the Boltzmann equation for the hard-sphere model, Arch. Ration. Mech. Anal., 173 (2004), pp. 279-296.

[20] HA, S.-Y., LEE, H., Global well-posedness of the relativistic Vlasov-Yukawa system with small data, J. Math. Phys., 48 (2007), 123508.

[21] Hejtmanek, J., Scattering theory of the linearized Boltzmann equation, Comm. Math. Phys., 43 (1975), pp. 109-120.

[22] Illner, R., Shinbrot, M., The Boltzmann equation, global existence for a rare gas in an infinite vacuum, Comm. Math. Phys., 95 (1984), pp. 217-226.

[23] Hauray, M., Jabin, P.-E., N-particles approximation of the Vlasov equations with singular potential, Arch. Ration. Mech. Anal., 183 (2007), pp. 489-524.

[24] Kaniel, S., Shinbrot, M., The Boltzmann equation 1. Uniqueness and local existence, Comm. Math. Phys., 58 (1978), pp. 65-84.

[25] Lax, P. D., Phillips, R. S., Scattering theory. Second edition. With appendices by Cathleen S. Morawetz and Georg Schmidt. Pure and Applied Mathematics, 26. Academic Press, Inc., Boston, MA, 1989.

[26] Lu, X., Spatial decay solutions of the Boltzmann equation: converse properties of long time limiting behavior, SIAM J. Math. Anal., 30 (1999), pp. 1151-1174.

[27] Mucha, P. B., Global existence for the Einstein-Boltzmann equation in the flat RoberstonWalker space-time, Comm. Math. Phys., 203 (1999), pp. 107-118.

[28] Mucha, P. B., Global existence of solutions of the Einstein-Boltzmann equation in the spatially homogeneous case, Evolution equations: existence, regularity and singularities, Warsaw 1998, 175-180, Banach Center Publ., 52, Polish Acad. Sci., Warsaw, 2000.

[29] Perthame, B., Time decay, propagation of low moments and dispersive effects for kinetic equations, Comm. Partial Differential Equations, 21 (1996), pp. 659-686.

[30] Rein, G., Collisionless kinetic equations from astrophysics: The Vlasov-Poisson system in Handbook of Differential Equations, Evolutionary Equations, 3 (2007), pp. 393-476.

[31] Reed, M., Simon, B., Methods of modern mathematical physics, Vol III, New York, Academic press.

[32] Polewczak, J., Classical Solution of the nonlinear Boltzmann equation in all $\mathbb{R}^{3}$ : Asymptotic behavior of solutions, J. Stat. Phys., 50 (1988), pp. 611-632.

[33] Simon, B., Existence of the scattering matrix for the linearized Boltzmann equation, Comm. Math. Phys., 41 (1975), pp. 99-108.

[34] Strauss, W., Nonlinear scattering theory in "Scattering theory in mathematical physics in the Proceedings of the NATO Advanced Study Institute", 1973 /ed. by J.A. Lavita and J.-P. Marchand. Dordrecht ; Boston : Reidel, 1974.

[35] Toscani, G., H-thoerem and asymptotic trend of the solution for a rarefied gas in a vacuum, Arch. Rational Mech. Anal., 100 (1987), pp. 1-12.

[36] Yukawa, H., On the interaction of elementary particles, Proc. Phys.Math. Soc. Japan, 17 (1935), pp. 48-57. 